\title{
Controversies in acetaminophen nephrotoxicity
}

\author{
Woo Yeong Park \\ Department of Internal Medicine, Keimyung University School of Medicine, Keimyung University Kidney Institute, Daegu, Republic of \\ Korea
}

Acetaminophen is one of the most common and available analgesic and antipyretic medications worldwide and is highly accessible because it has fewer side effects than any other analgesic or antipyretic. However, acetaminophen overdose can cause serious and lethal hepatotoxicity. The daily maximal dose of acetaminophen is $4.0 \mathrm{~g}$, and excessive doses between $7.5 \mathrm{~g}$ and $10.0 \mathrm{~g}$ can cause hepatotoxicity [1] because about $10 \%$ of acetaminophen is metabolized into the toxic metabolite $\mathrm{N}$-acetyl$p$-benzoquinone imine (NAPQI) by cytochrome P450 [2] Large doses of acetaminophen result in severe glutathione depletion with overproduction of NAPQI, causing hepatotoxicity.

With similar pathophysiology, concurrent nephrotoxicity induced by acetaminophen has been raised as a concern over the past few decades $[3,4]$. However, regarding kidney function, acetaminophen use with appropriate therapeutic doses should be safer than nonsteroidal antiinflammatory drugs (NSAIDs) or opiates. The National Kidney Foundation (NKF) recommends a single dose of acetaminophen as the drug of choice for pain control in

Received February 13, 2020; Revised February 23, 2020;

Accepted February 24, 2020

Edited by Gheun-Ho Kim, Hanyang University, Seoul, Republic of Korea

Correspondence: Woo Yeong Park

Department of Internal Medicine, Keimyung University School of Medicine, Keimyung University Kidney Institute, 1035 Dalgubeoldaero, Dalseo-gu, Daegu 42601, Republic of Korea. E-mail: parkwy2015@dsmc.or.kr

Copyright @ 2020 by The Korean Society of Nephrology

(a) This is an open-access article distributed under the terms of the Creative Commons Attribution Non-Commercial License (http://creativecommons. org/licenses/by-nc-nd/4.0/), which permits unrestricted non-commercial use, distribution, and reproduction in any medium, provided the original work is properly cited. patients with kidney disease [5].

In comparison, ingestion of toxic amounts of acetaminophen can cause acute kidney injury (AKI) because of acute tubular necrosis at the proximal tubule, and habitual acetaminophen use may result in chronic kidney disease (CKD) and end-stage renal disease (ESRD) from chronic interstitial fibrosis or papillary necrosis [6]. Excessive amounts of acetaminophen and NAPQI are excreted by the kidney and may contribute to AKI. Furthermore, the associated oxidative stress can lead to direct nephrotoxicity [7].

However, evidence that habitual use of acetaminophen alone increases the risk of ESRD has not been clearly demonstrated. A population-based cohort study reported that single-substance acetaminophen may be safe for patients with advanced CKD at stage 4 to 5 without an adverse effect on progression of CKD [8]. Finally, the association between acetaminophen use and renal impairment is still controversial.

In this issue of Kidney Research and Clinical Practice, Kanchanasurakit et al [9] performed a systematic review and meta-analysis for the association between acetaminophen and renal impairment. Of 13,097 articles identified, 5 (two cohort studies and three casecontrol studies) with 13,114 participants were included. All included studies were observational. Three studies focused on the association between acetaminophen use and CKD, another focused on AKI, and the other focused on AKI and CKD. The authors analyzed causes of heterogeneity based on study design (case-control and cohort studies), identified publication bias, and discussed study limitations. More than one tool was used in the analyses to provide confidence in evaluation of the risk of bias in the observational studies. The results concluded that acetaminophen users without a prior history of kidney 


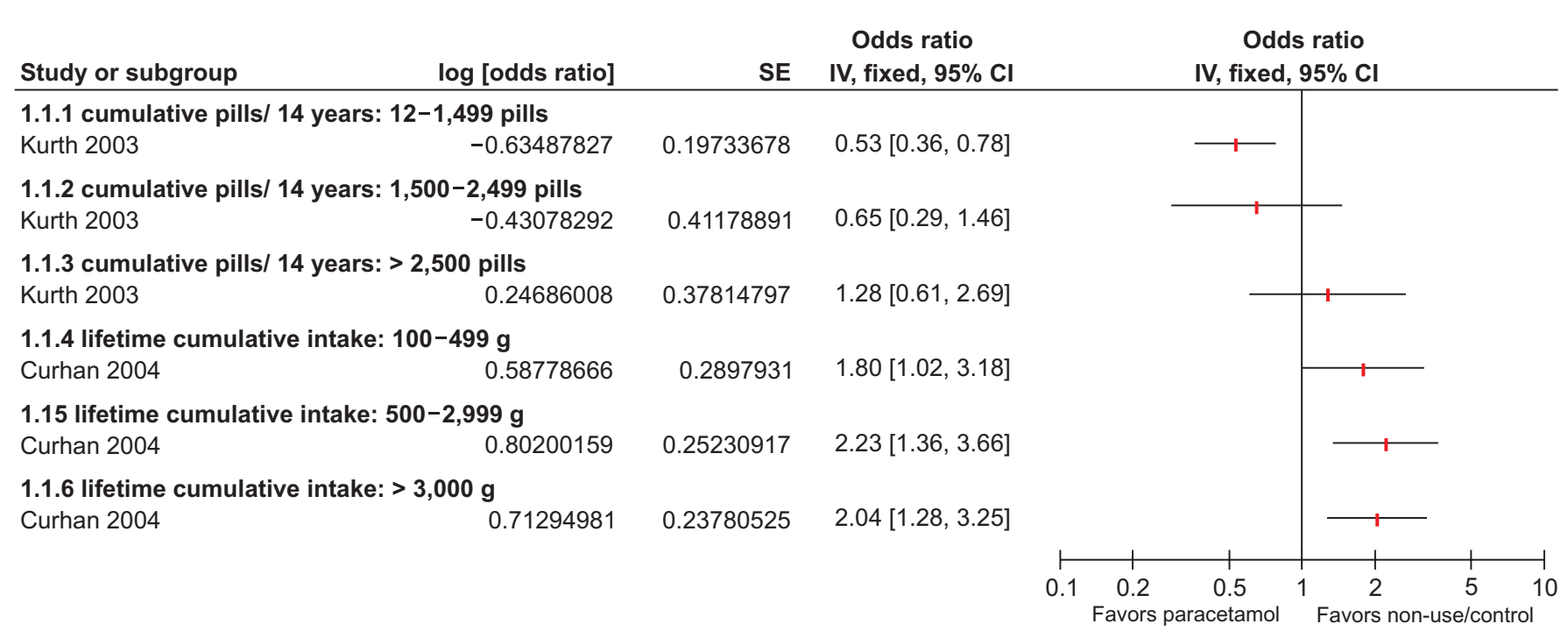

Figure 1. Odd ratios of decrease in estimated glomerular filtration rate of at least $30 \mathrm{~mL} / \mathrm{min} / 1.73 \mathrm{~m}^{2}$ in patients taking versus not taking acetaminophen. Adapted from the article of Roberts et al [10].

$\mathrm{Cl}$, confidence interval; IV, intravenous; SE, standard error.

disease had a significantly increased risk $(31 \%)$ of renal impairment compared with non-users (adjusted odds ratio, 1.31; 95\% confidence interval, 1.10-1.57). Therefore, attention should be paid to use of acetaminophen in patients with risk factors of nephrotoxicity.

In the current study, risk factors for acetaminophen nephrotoxicity were evaluated according to dose (therapeutic dose $\leq 4 \mathrm{~g}$ in 24 hours vs. toxic dose $>4 \mathrm{~g}$ in 24 hours), administration duration (regular therapeutic doses $\leq 30$ days vs. $>30$ days; $<15$ doses/month vs. $>15$ doses/month; lifetime cumulative dose $0.1-99.9 \mathrm{~g}$ vs. $100-$ $999.9 \mathrm{~g}$ ), time course of toxic dose (acute $>4 \mathrm{~g}$ in 1 hour vs. chronic $>4 \mathrm{~g}$ daily for 3-4 days or lifetime cumulative dose $>1,000 \mathrm{~g}$ ), type of renal impairment (AKI, CKD, or both), prior exposure to other potentially nephrotoxic drugs, and comorbidity (e.g., cardiovascular disease, hypertension, and diabetes mellitus). In conclusion, the identified risk factors were toxic dose, acute exposure, cumulative lifetime dose $>1,000 \mathrm{~g}$ of acetaminophen, comorbidities (including diabetes mellitus and liver failure), alcohol intake, and concomitant use of nephrotoxic drugs.

There were some limitations to this study. No randomized controlled trials (RCTs) were included in this analysis, and inherent biases could not be avoided. Performance of RCTs is not easy because of ethical issues around use of a placebo in patients with pain. In addition, the criteria of renal impairment were not uniform and not clearly defined. Thus, there is a possibility that the relationship between acetaminophen and renal impairment may be distorted, and there is need for caution in interpretation and application of the results.

Nevertheless, acetaminophen is probably the most frequent prescription among nephrologists to relive pain in CKD patients compared to other analgesics such as NSAIDs or opioids. Unlike NSAIDs, it is possible that renal aggravation is not common in patients taking acetaminophen. The resultant association between acetaminophen use and renal impairment may exist because acetaminophen is very frequently prescribed for CKD patients. However, physicians should consider the possibility that acute or long-term overdose of acetaminophen may cause nephrotoxicity, increasing risk of kidney failure, especially in patients with comorbidities or concomitant use of nephrotoxic drugs. Figure 1 shows that lifetime cumulative dose $>100 \mathrm{~g}$ was associated with a decrease in estimated glomerular filtration rate of at least $30 \mathrm{~mL} / \mathrm{min} / 1.73 \mathrm{~m}^{2}$ [10]. Therefore, intermittent short-term use of an appropriate dose is recommended in patients with risk factors, especially those with kidney disease.

\section{Conflicts of interest}

The author has no conflicts of interest to declare. 


\section{References}

[1] Zed PJ, Krenzelok EP. Treatment of acetaminophen overdose. Am J Health Syst Pharm 1999;56:1081-1091.

[2] Moyer AM, Fridley BL, Jenkins GD, et al. AcetaminophenNAPQI hepatotoxicity: a cell line model system genomewide association study. Toxicol Sci 2011;120:33-41.

[3] Perneger TV, Whelton PK, Klag MJ. Risk of kidney failure associated with the use of acetaminophen, aspirin, and nonsteroidal antiinflammatory drugs. N Engl J Med 1994; 331:1675-1679.

[4] Fored CM, Ejerblad E, Lindblad P, et al. Acetaminophen, aspirin, and chronic renal failure. N Engl J Med 2001;345: 1801-1808.

[5] Henrich WL, Agodoa LE, Barrett B, et al. Analgesics and the kidney: summary and recommendations to the Scientific Advisory Board of the National Kidney Foundation from an
Ad Hoc Committee of the National Kidney Foundation. Am J Kidney Dis 1996;27:162-165.

[6] Blantz RC. Acetaminophen: acute and chronic effects on renal function. Am J Kidney Dis 1996;28(1 Suppl 1):S3-S6.

[7] Mazer M, Perrone J. Acetaminophen-induced nephrotoxicity: pathophysiology, clinical manifestations, and management. J Med Toxicol 2008;4:2-6.

[8] Evans M, Fored CM, Bellocco R, et al. Acetaminophen, aspirin and progression of advanced chronic kidney disease. Nephrol Dial Transplant 2009;24:1908-1918.

[9] Kanchanasurakit S, Arsu A, Siriplabpla W, et al. Acetaminophen use and risk of renal impairment: a systematic review and meta-analysis. Kidney Res Clin Pract 2020;39:81-92.

[10] Roberts E, Delgado Nunes V, Buckner S, et al. Paracetamol: not as safe as we thought? A systematic literature review of observational studies. Ann Rheum Dis 2016;75:552-559. 